\title{
ANÁLISIS DE LA OBRA MUSICAL SELECCIONADA DE CUATRO COMPOSITORES OCAÑEROS
}

\section{ANALYSIS OF THE SELECTED MUSICAL WORK OF FOUR COMPOSERS FROM OCAÑA}

\author{
Esp. Juan Pablo Peñaranda Franco ${ }^{a}$, Esp. José Alejandro Navarro Claro ${ }^{\mathrm{b}}$
}

\author{
${ }^{a}$ Grupo de Investigación de la Facultad de Educación, Artes y Humanidades GIFEAH, \\ Universidad Francisco de Paula Santander Ocaña,Vía Acolsure, Sede el Algodonal, Ocaña, \\ Colombia, \\ jppenarandaf@ufpso.edu.co \\ ${ }^{\mathrm{b}}$ Grupo de Investigación de la Facultad de Educación, Artes y Humanidades GIFEAH, \\ Universidad Francisco de Paula Santander Ocaña, Vía Acolsure, Sede el Algodonal, Ocaña, \\ Colombia, janavarroc@ufpso.edu.co
}

Fecha de recepción: 01-12-2016

Fecha de aprobación: 17-05-2017

\begin{abstract}
Resumen: El presente artículo presenta el análisis realizado a las obras musicales seleccionadas de los compositores ocañeros homenajeados en el catálogo musical "Ocaña: legado de música, letras y pasiones", el cual fue producto de la ejecución del proyecto "Compilación, análisis, clasificación y estudio de la obra musical de 4 compositores de Ocaña y la provincia; y elaboración de su catálogo musical", con el objeto de rescatar la identidad musical y evitar la pérdida de la memoria cultural y musical de la región en peligro de desaparecer. El proyecto investigativo es de tipo cualitativo y el objeto de estudio fue la obra musical de los autores Rafael Contreras Navarro, Carlos Julio Melo Paredes, Carmen Noel Paba y Carlos Guillermo Lemus Sepúlveda. Como resultado del mismo, se realizó un análisis musical de las 2 obras de cada compositor que fueron seleccionadas, arregladas y producidas musicalmente en diferentes formatos instrumentales para el disco del catálogo. De este análisis se evidencia que en cada uno de ellos se siguen en términos generales los criterios de composición de la época reflejado en las métricas, los ritmos, los tonos.
\end{abstract}

Palabras clave: folklore, memoria musical, música, patrimonio inmaterial.

Abstract This article presents the analysis made to the selected musical works of the composers from Ocaña who was honored in the musical catalog "Ocaña: legacy of music, letters and passions", which was the result of the execution of the project "Compilation, analysis, 
classification and study of the musical work of 4 composers from Ocaña and the Provincia and its musical elaboration ", in order to rescue the musical identity and to avoid the loss of cultural and musical memory of the region in danger of disappearing. The research project is qualitative type and the object of study was the musical work of the composers Rafael Contreras Navarro, Carlos Julio Melo Paredes, Carmen Noel Paba and Carlos Guillermo Lemus Sepúlveda. As a result of this, a musical analysis of the 2 works of each composer, those were arranged and produced musically in different instrumental formats for the disc of the catalog. From this analysis, it's evident that in each one of them, the composition criteria of their time are followed, because the metric, the rhythms, the tones, etc. reflect that.

Keywords: Folklore, musical memory, music, intangible heritage.

\section{INTRODUCCIÓN}

La música es una manifestación cultural que encuentra en la vida de los pueblos un puesto privilegiado. Es innegable que la misma interviene en el desarrollo emocional e intelectual de los individuos. El lenguaje musical es el lenguaje propio del espíritu humano. Cada región vive el florecimiento de este arte, por ello, en Ocaña, hay una vasta tradición musical; son muchos los intérpretes y compositores que han surgido en la región. De allí que Santafé (2009) escribe:

Desde su misma génesis, dio Ocaña muestras de ser una ciudad con vocación cultural, siendo la música y la poesía las dos manifestaciones de mayor relevancia. Fueron los curas encomenderos y los padres que regentaron colegios y escuelas, quienes se dieron a la tarea de enseñar la música religiosa y profana, en un ambiente que les fue fácil por las condiciones del clima, la sociedad y la familia, y porque encontraron el elemento humano capaz y apto para el goce de la belleza auditiva (Pág. 66)

Ante la propia historia, se corre el riesgo del olvido, en caso de no existir un ejercicio que registre los acontecimientos y sentires propios sea cual fuere la realidad que se intente datar.
En la provincia de Ocaña, con un legado histórico bastante notable incluso desde la época colonial, se han hecho algunos intentos por registrar los principales referentes en el campo musical como es el caso de los libros "Aires de mi tierra: apuntes para la historia musical de Norte de Santander" de Rafael Santafé; "Biografías musicales anecdotadas" de Alonso Bayona Quintero; "Banda Municipal de Ocaña, su historia, su fundación y bandas que le precedieron" de Élmer Paba Castro; "Lecturas de música Colombiana" de Rafael Contreras. Algunos artículos de Gabriel Ángel Páez Téllez y los intentos académicos de la Academia de historia de Ocaña entre otros que podrían contarse facilmente, por ser muy pocos. Si los relatos de vida corren el riesgo de perderse, ¿qué pensar de las obras como tal? ¿De la construcción escrita de cada uno de estos autores, que por ser únicamente legado para músicos es más frágil por no ser estimada por la población en general?

Algunos de los músicos destacados durante el siglo XX en Ocaña fueron: el maestro Rafael Contreras Navarro, compositor del inmortal bambuco "Ocañerita"; el maestro Carlos Julio Melo Paredes, nacido en el corregimiento de Buenavista y compositor de la danza "Las Américas"; el maestro Miguel Ángel Pino, a quien se le conocen obras como "Aromas" y "No te vas" (con letra de Adolfo Milanés); el 
maestro Carlos Guillermo Lemus Sepúlveda, conocido como "El Caballero de la Música", nacido en el corregimiento de Otaré, que llegó a ser el director de la Banda Municipal de Ocaña durante más de una década, dejando una extensa lista de composiciones entre las que se encuentran: el pasillo "Aromas de la Tarde", el vals "Sueño con mi madre"; y los bambucos "Otaré" y “Tejarito". Otros músicos representativos de Ocaña y su provincia fueron: Carmen Noel Paba Forero "Carmito" y Gilberto Núñez Sarmiento, ambos nacidos en el municipio de Convención, Nemesio Pino Navarro y Fray Campo Elías Claro Carrascal. Este último nacido en el municipio de La Playa de Belén (Santafé, 2009).

Este proyecto de investigación es una iniciativa de la Fundación Cinco Sentidos en alianza con la Universidad Francisco de Paula Santander de Ocaña y el Ministerio de Cultura; a fin de rescatar y fortalecer toda la riqueza musical que ha caracterizado a esta región. Para ello se realiza una investigación que tuvo como objetivo elaborar un catálogo de la obra musical de cuatro compositores de Ocaña y la provincia: Rafael Contreras Navarro, Carlos Julio Melo Paredes, Carmen Noel Paba Forero, y Carlos Guillermo Lemus Sepúlveda.

\section{METODOLOGÍA}

El proyecto investigativo estuvo enmarcado en el trabajo "Ocaña, legado de música, letras y pasiones" producido por el "Grupo de Investigación de la Facultad de Educación, Artes y Humanidades (GIFEAH)" que tuvo enfoque cualitativo en el cual, el centro del proceso se concretó en el enfoque de historia de vida; técnica que permite ahondar en los registros de vida de un individuo. El objeto de estudio del mismo se configuró en la obra musical de los autores Rafael Contreras, Carlos Julio Melo Paredes, Carmen Noel Paba y Carlos Guillermo Lemus Sepúlveda. Este trabajo tuvo dos componentes: el primero la recopilación de la historia de vida de cada uno de los autores y el segundo, sobre el que versa este artículo, la selección de dos obras representativas de cada uno de los autores y el rescate de las mismas para realizar el arreglo musical pertinente para mediar la grabación de un disco conmemorativo.

Una base para poder acudir a registros confiables fue el reconocimiento de las obras frente a sus compositores, por parte de la comunidad de músicos de la provincia de Ocaña en primera instancia y por la naturaleza de las obras -el ser en un grado $\mathrm{u}$ otro del dominio público-; incluso por la comunidad Ocañera en general. Así mismo, al recurrirse a la familia misma y a las amistades de los compositores se cuidó la veracidad de la información que se recopiló y sirvió de sustento para construir el catálogo. Se buscaron registros realizados por el puño y letra de los compositores como fuentes de primera mano, evitando de esta forma, posibles derivaciones.

Para tal fin no solo las partituras escritas fueron suficientes sino que hubo necesidad incluso de algunas piezas grabadas pues fue difícil poder acceder al registro escrito de todas las piezas debido a que los arreglos musicales suelen escribirse con un score, es decir un documento en el que se ubican las partituras de todos los instrumentos para las que se compone el arreglo; por ser obras para bandas musicales, a cada músico se le entrega su partitura. Esto complica el asunto debido a que en algunos casos la melodía se interpreta por ciertos instrumentos de la banda mientras la armonía se delega sobre todo a los instrumentos construidos para tal fin. La recopilación de las piezas musicales se dificulta pues no se contó con los scores completos, sino que se podían ubicar apenas pedazos, o bien de melodía o de armonía. Poder contar con algunas grabaciones se hizo por ello más importante de lo que podría presuponerse. 
Para escoger las obras, se tuvo la necesidad de acudir a la comunidad de músicos de la provincia. Se les dio a escoger a una muestra significativa de 5 integrantes - escogidos de forma aleatoria- de la desintegrada banda municipal, en la que los cuatro maestros escogidos fueron integrantes e incluso directores. Esto para garantizar que las obras fueran las más representativas de cada uno de los maestros que fueron objeto de estudio.

Una vez recogidas la obras, la tarea consistió en la recolección de todo el material que pudiese haber sobre cada una de ellas con el cuidado de utilizar aquellas que preferentemente estuviesen en el conjunto de obras que los maestros de la banda municipal aún conservan, pues ello es garantía de haber sido escritas por el mismo puño y letra de cada uno de los compositores.

Igualmente los cuadernillos musicales que los familiares pudieran conservar entre sus haberes y las grabaciones de algunos temas. Con los fragmentos de algunas obras escritas recogidas, se procedió a comparar las mismas con los audios para corroborar que se desarrollaran en el mismo tono original escrito. Los fragmentos incompletos se completaron y se hicieron arreglos por parte de Juan R. Forero y Orlando Pérez Rosso, productor musical ocañero encargado de la grabación del disco, proceso que se llevó a cabo en los estudios profesionales de Audiovisión en la ciudad de Bogotá.

\section{RESULTADOS}

\section{Rafael Contreras Navarro}

Romero, Retavisca, \& Guevara refieren que hablar de Rafael Contreras Navarro es referirse al ícono de la música tradicional en la provincia Ocañera, por ser principal precursor del arte en la región. Músico, político, columnista y amante de la literatura (S.A.); escribe un sin número de obras entre las cuales, muy representativas son:

\section{Ocañerita}

Bambuco que pasó por una serie de transformaciones en su nombre. Inicialmente denominado "Morales Berti" y posteriormente "Barbatuscas" es la obra insignia del compositor ocañero debido a que con la adaptación de la letra por parte de Miguel Ángel Quintero Pacheco, poeta ocañero, se convierte en himno folklórico del municipio de Ocaña (Romero, Retavisca, \& Guevara, S.A.).

Este bambuco tiene un arreglo de Orlando Pérez Rosso para cinco instrumentos: violín primero y segundo, viola, violonchelo y contrabajo. La melodía del mismo está pensada para ser interpretada de forma oral.

La métrica de esta obra conserva las características de la métrica de los bambucos escritos en su tiempo: 3/4. Este simple hecho nos ubica en la garantía que la obra fue compuesta por el maestro pues la usanza actual es una métrica de 6/8. Este hecho hace contrastar a los bambucos nacidos en la actualidad y los escritos en épocas anteriores debido a la diferencia de los acentos que ambas métricas promueven.

La obra está compuesta con una armadura de sol menor y se realizan varias modulaciones en el desarrollo de la obra hacia la relativa mayor, es decir, sol mayor. Esto confirma otra tendencia de los compositores de antaño, la preferencia por establecer modulaciones teniendo como base las relativas mayores y menores. Esta obra, por lo tanto no es la excepción a esa tendencia si bien, generalmente se establecen tres modulaciones pues generalmente las obras terminan con el mismo tono con que comenzaron, en esta obra se desarrolla una serie de tres modulaciones concluyéndose la misma en sol mayor. En total 
son 160 compases cargados de los rasgos más sentidos del vivir ocañero.

\section{En Lontananza}

Danza con métrica de 3/4 armada aparentemente en fa con tonalidad real de mi bemol mayor. Tiene una modulación a do mayor en el compás 30 y luego hay un segundo cambio de tono en el compás 54 a la bemol mayor. Esta obra presenta una estructura muy poco usual debido a que los cambios de tono en la construcción de la misma no refieren al esquema de uso de las relativas mayores o menores de la época.

Sin embargo, lo que sí es característico, es el hecho de ser una danza pues en la época se componían muchas de ellas para ser interpretadas por los colectivos de las bandas de la región.

En este caso, el arreglo de esta obra musical fue realizado por Juan R. Forero para ser interpretado por un cuarteto de saxofones (soprano, alto, tenor y barítono).

\section{Carmen Noel Paba Forero}

Durán, Ropero, \& Márquez refieren que el Maestro Carmen Noel Paba, oriundo del municipio de Convención, nace el 4 de septiembre de 1931 de la familia conformada por los señores Anastasio Paba y Teófila Forero. Entre los aires que compuso se encuentran los pasillos y los pasodobles. Los mismos nutrieron la galería de obras de diversas bandas municipales de diversos municipios (S.A.).

Dos obras representativas del maestro son:

\section{Saltarín}

Pasillo construido con una métrica de 3/4 que contiene en este primer elemento según Abadía las propiedades clásicas del pasillo, ser un ritmo para propiciar que en el baile, el artista deba dar pequeños pasos; de allí proviene su nombre (1973). Incluso el título de este pasillo es evocador de esta realidad.

En este caso particular se puede evidenciar una modulación en ciclos alternando de un tono inicial de fa mayor hacia si bemol mayor reiteradamente, tres veces hasta concluir el tema en si bemol mayor.

Los arreglos de este tema fueron realizados inicialmente para un cuarteto de clarinetes por Gustavo Mantilla, posteriomente Orlando Pérez Rosso hizo una adaptación para cuarteto de saxofones, versión que fue grabada en el disco, con el mismo formato utilizado en el tema "En Lontananza" del maestro Rafael Contreras Navarro: saxofón soprano, alto, tenor y barítono.

\section{Luz Elena}

Nuevamente el maestro Carmen Noel incursiona en el terreno del pasillo con su métrica característica de 3/4. En este caso una obra cuya extensión aparente no es tal, sino que recurre a la repetición reiterada de compases para brindarle mayor duración. Acompasado por un piano y un clarinete en Mi $b$, el arreglo de Juan R. Forero, nos muestra un virtuosismo con una sencillez datada incluso en la única modulación de todo el tema, ya que empieza por sol mayor para concluir en do mayor.

\section{Carlos Julio Melo}

Sánchez, Villareal, \& Higuera mencionan que el maestro nació en Buenavista de la familia conformada por el señor Francisco Antonio Melo y Luciana Paredes el 17 de abril del año 1905; como dato curioso, el día de su nacimiento estuvo pasado por una gran tempestad. Proviene de una famosa familia de músicos Francisco Melo intérprete del tiple y Julio Melo, su tío, interpretaba la 
guitarra; e incluso sus dos hermanos Rafael y especialmente Pedro Antonio Melo que lo acompañó en algunos proyectos musicales (S.A.).

Entre sus composiciones se encuentran:

\section{Las Américas}

Hermosa danza con métrica en 2/4 cuyo arreglo fue realizado por Orlando Pérez Rosso. La letra es de autoría del mismo maestro Carlos Julio Melo expresando con ello la variedad de facetas del compositor. La pieza es una obra escrita en sol menor que hace tres cambios de tono: en el compás 54 modula a sol mayor y en el 83 a sol menor para finalizar en el compás 105 cambiando a sol mayor nuevamente.

Este esquema corrobora la antigua usanza de cambios de tono. Esta pieza está compuesta para piano, saxofón alto, saxo tenor, barítono y fliscorno. El aspecto melódico es asumido por la voz, con letras evocadoras de paisajes americanos, de la valentía de los nacidos en su tierra y el regocijo de asumir la raza americana.

\section{Flor de Ocaña}

Hermosa guabina escrita con una métrica de 3/4. Difiere de todas las obras en un aspecto particular: escrita sin modulaciones pues toda la obra conserva un tono en $\mathrm{Re}$ menor. Los arreglos de esta obra para ser grabados en el disco fueron realizados por Juan R. Forero. El arreglo de la misma está escrito para flauta, chelo y piano.

\section{Carlos Guillermo Lemus Sepúlveda}

Carlos Guillermo Lemus Sepúlveda, más conocido como 'Memo', nació en Otaré, corregimiento de Ocaña (Norte de Santander) el 10 de febrero de 1924. "Sus padres Tiberio Lemus y Leonor Sepúlveda inculcaron en él y sus seis hermanos, los valores que hicieron de "Memo" un hombre honesto y prudente". (Sánchez, Machi, \& Sanjuán, S.A.)

El maestro Orlando Alfonso Velásquez Rincón, refiriéndose al maestro Lemus expresó:

Los ocañeros debemos sentirnos
privilegiados de contar con un talento
como el del Maestro "Memo" como
cariñosamente le llamamos y debemos
dar gracias al Creador por habernos
escogido para vivir en su misma época y
deleitar nuestro espíritu con su música
(Santafé, S.A.)

Las composiciones escogidas de este insigne maestro son:

\section{Antañares}

Bello pasillo arreglado por Orlando Pérez Rosso para clarinete en mib, violín I y II; viola y violonchelo. Esta pieza comienza con un tono de Mi bemol mayor y modula a la bemol mayor; cosa no muy común en obras escritas en estos maestros en cuyas obras se ven modulaciones asociadas a las relativas mayores y menores. Conserva la métrica en 3/4 característica de los pasillos.

\section{Ledy Esperanza}

Pasillo escrito en si bemol mayor que conserva el mismo tono en toda la obra. Con arreglos de Juan R. Forero para clarinete en mib y piano cierra de forma magistral el conjunto de ocho obras escogidas para hacer parte de este catálogo musical producido.

\section{CONCLUSIONES.}

Este tipo de proyectos investigativos permite conocer y rescatar el legado musical de los grandes compositores de la Provincia de 
Ocaña ante la amenaza del olvido paulatino de su herencia por parte de las nuevas generaciones. El escenario que pudo constatarse con el proyecto investigativo fue desalentador pues las dificultades que aparecieron en el curso del mismo reflejaron el desinterés de las instituciones y habitantes de la región por preservar este legado.

Se logró rescatar con este proyecto la obra más representativa de estos cuatro compositores y con ello se impactó de manera positiva el imaginario no sólo de los familiares sino de la comunidad en general; es necesario por ello seguir en este propósito pues el número de compositores de la región excede el número de maestros en los que se centró la investigación realizada.

Uno de los factores que incide para que la pérdida de memoria histórica en torno al universo musical de la provincia es la falta de documentación, de poner por escrito el legado artístico que va generándose día a día con las nuevas generaciones de cultores para establecer la conexión necesaria entre el pasado y el presente; siendo el pasado el cincel que determina las acciones que los pueblos van construyendo para su futuro. Ello hace necesario que se emprendan labores historiográficas para rescatar las huellas dejadas atrás y así mismo la emergencia de cronistas que vayan recopilando el legado que se va edificando.

Se constató que hay una línea común de ritmos musicales entre los que el pasillo fue el ritmo más compuesto por los maestros objeto de investigación.

\section{FINANCIACIÓN}

Se hace necesario mencionar que el proyecto de investigación nace del proyecto denominado "Compilación, análisis, clasificación y estudio de la obra musical de cuatro compositores de Ocaña y al Provincia; y elaboración de su catálogo musical" presentado por la "Fundación Cinco Sentidos" en el año 2012 al "Programa Nacional de Concertación del Ministerio de Cultura" para ser aprobado por el mismo en el año 2013.

Con este precedente, la "Universidad Francisco de Paula Santander Ocaña" se une a la iniciativa por medio del "Grupo de Investigación de la Facultad de Artes y Humanidades (GIFEAH)"

\section{BIBLIOGRAFÍA}

Abadía, G. (1973). La música folklórica Colombiana. Bogotá: Universidad Nacional de Colombia.

Claro, J. (31 de Enero de 2003). Alirio Gómez Picón. Recuperado el 21 de Noviembre de 2016, de http://laplayadebelen.org/JAIRO_CLAR O/ALIRIO_GOMEZ_PICON.html

Clavijo, C. (2015). Carlos Julio Melo. (W. Higuera, \& J. Villareal, Entrevistadores)

Contreras, M. (2015). Rafael Contreras Navarro. (L. Retavisca, \& L. Guevara, Entrevistadores)

Durán, C., Ropero, S., \& Márquez, M. (S.A.). Carmen Noel Paba Forero: un apasionado por la música y la 
composición. En UFPSO, Ocaña:

legado de música, letras y pasiones

(págs. 36-49). Ocaña: S.E.

García, É. (2013). Carlos Guillermo Lemus. (L. Machi, \& A. Sanjuán, Entrevistadores)

Lemus, L. F. (2013). (L. Machi, \& A. Sanjuán, Entrevistadores)

Romero, L., Retavisca, L., \& Guevara, M.

(S.A.). Rafael Contreras Navarro: ícono de la música tradicional de la provincia de Ocaña. En UFPSO, Ocaña: legado de música, letras y pasiones (págs. 1535). Ocaña: S.E.

Sánchez, E., Machi, L., \& Sanjuán, A. (S.A.). Ocaña: legado de música, letras y pasiones. Ocaña: S.E.

Sánchez, E., Villareal, T., \& Higuera, W. (S.A.). Carlos Julio Melo: un hombre cautivado por los deseos del alma y de la música. En UFPSO, Ocaña: legado de música, letras y pasiones (págs. 50-71). Ocaña: S.E.

Sánchez, M. (2015). Carlos Julio Melo. (W. Higuera, \& J. Villareal, Entrevistadores)

Santafé, R. (S.A.). Aires de mi tierra: apuntes para la historia musical de Norte de Santander. S.C.: S.E. 